Monika Albrecht / Dirk Göttsche (Hrsg.) Bachmann-Handbuch 
Monika Albrecht /

Dirk Göttsche (Hrsg.)

\section{Bachmann- Handbuch}

Leben - Werk - Wirkung 
Die Deutsche Bibliothek - CIP-Einheitsaufnahme

Bachmann-Handbuch :

Leben - Werk - Wirkung /

Monika Albrecht/Dirk Göttsche (Hrsg.).

- Stuttgart ; Weimar : Metzler, 2002

ISBN 978-3-476-01810-6

ISBN 978-3-476-01810-6

ISBN 978-3-476-05201-8 (eBook)

DOI 10.1007/978-3-476-05201-8

Dieses Werk einschließlich aller seiner Teile ist urheberrechtlich geschützt. Jede Verwertung außerhalb der engen Grenzen des Urheberrechtsgesetzes ist ohne Zustimmung des Verlages unzulässig und strafbar. Das gilt insbesondere für Vervielfältigungen, Übersetzungen, Mikroverfilmungen und die Einspeicherung und Verarbeitung in elektronischen Systemen.

(C) 2002 Springer-Verlag GmbH Deutschland

Ursprünglich erschienen bei J.B. Metzlersche Verlagsbuchhandlung

und Carl Ernst Poeschel Verlag GmbH in Stuttgart 2002

www.metzlerverlag.de

Info@metzlerverlag.de 


\section{Inhaltsverzeichnis}

\section{Vorwort VII}

\section{Grundlagen 1}

1. Leben und Werk im Überblick - eine Chronik 2

2. Rezeptionsgeschichte 22

2.1. Rezeptionsgeschichte zu Lebzeiten 22

2.2. Rezeptionsgeschichte seit Bachmanns Tod 26

2.3. Literarische Rezeption 35

3. Editionsgeschichte und Nachlaß 42

\section{Das Werk 47}

1. Jugendwerke 48

2. Lyrik 53

2.1. Frühe Gedichte 53

2.2. Die gestundete Zeit 57

2.3. Anrufung des großen Bären und Gedichte aus dem Umfeld 67

2.4. Späte Gedichte 78

3. Hörspiele 83

4. Libretti 97

5. Erzählprosa 105

5.1. Frühe Erzählprosa 105

5.2. Das dreißigste Jahr und Erzählfragmente aus dem Umfeld 112

5.3. Todesarten-Projekt 127

5.3.1. Überblick 127

5.3.2. Malina 130

5.3.3. Das Buch Franza 144

5.3.4. Andere unvollendete TodesartenTexte 152

5.4. Simultan und Erzählfragmente aus dem Umfeld 159

6. Künstlerische und journalistische Prosa 172

7. Kritische Schriften 184

7.1. Philosophische Essays und Dissertation 184

7.2. Musikästhetische Essays 188

7.3. Literaturkritische Essays und Frankfurter Vorlesungen 191

8. Übersetzungen 204

\section{Kontexte und Diskurse in} Bachmanns Werk 211

1. Bachmann und die Philosophie 212

1.1. Existentialphilosophie und Existentialismus 212

1.2. Sprachphilosophie und poetologische Sprachreflexion 214

1.3. Kritische Theorie und Soziologie 216

1.4. Religion 218

1.5. Bachmanns Utopiebegriff 220

2. Psychologie, Psychoanalyse und Psychiatrie in Bachmanns Werk 223

3. Bachmann und die Zeitgeschichte 237

3.1. Nationalsozialismus 237

3.2. Die Entwicklung der Nachkriegsgesellschaft 246

3.3. Der kulturgeschichtliche Umbruch von 1968252

3.4. Postkolonialismus und Kritischer Exotismus 255

4. Literarische Kontexte, Dialoge und Lektüren 259

4.1. Deutschsprachige Literatur des 18. und 19. Jahrhunderts 259

4.2. Europäische Literatur vor 1900263

4.3. Klassische Moderne 270

4.4. Deutschsprachige Literatur nach 1945282

4.5. Bachmann und die 'Weltliteraturs ihrer Zeit 292

5. Bachmann und die Musik 297

\section{Anhang 309}

1. Siglenverzeichnis (mit Sigle zitierte Ausgaben) 310

2. Andere Ausgaben und Hilfsmittel 311

3. Ausgewählte Sekundärliteratur 312

3.1. Sammelbände 312

3.2. Monographien und Aufsätze 312

4. Werkregister 317

5. Personenregister 322

6. Mitarbeiter 329 


\section{Vorwort}

Nachdem die vierbändige Ausgabe der »Werke« (1978) erstmals einen zusammenhängenden Überblick über Ingeborg Bachmanns Werk und erste Einblicke in ihren literarischen Nachlaß ermöglicht hatte, ist es im Gefolge einer neuen feministischen Lektüre in den frühen 1980er Jahren geradezu zu einer »Bachmann-Rennaissance» gekommen (Stephan et al. 1987, S. 8), die bis heute anhält. Seither hat die Literaturwissenschaft eine Fülle neuer Erkenntnisse zu Ingeborg Bachmanns Werk hervorgebracht und zugleich grundlegend neue Perspektiven der Interpretation erarbeitet. Nach dem frühen Ruhm der Autorin als »neuer Stern am deutschen Poetenhimmel« (Blöcker) in den 1950er Jahren hat eine breite, lebhafte und vielschichtige Forschung in den vergangenen zwanzig Jahren so die Einschätzung Ingeborg Bachmanns als eine der wichtigsten deutschsprachigen Autorinnen der Nachkriegsjahrzehnte auf neuer Grundlage bekräftigt. Bachmanns Werk steht seitdem gleichbedeutend für seine Auseinandersetzung mit der - wie sie es nannte - »Krankheit unserer Zeit« (GuI, 72), mit der sozialen Gewalt der modernen westlichen Gesellschaft, mit dem verborgenen Zusammenhang zwischen patriarchalischer Gesellschaftsstruktur, katastrophischer Geschichte (Nationalsozialismus) und Unterwerfung bzw. Ausgrenzung des anderen (bis hin zum Neokolonialismus). Die fortdauernde Brisanz dieser Problemstellungen und die Reflektiertheit ihrer literarischen Darstellung sichern ihrem Werk zweifellos seine anhaltende Bedeutung.

Angesichts des wachsenden Abstands von der Entstehungszeit der Texte zeichnet sich seit einiger Zeit allerdings ein Forschungsumbruch $a b$, der die Begründung der fortdauernden Relevanz von Bachmanns Werk mit einer deutlicheren Historisierung im Sinne einer Neubewertung ihres Oeuvres im Kontext der literarischen Nachkriegsjahrzehnte verbindet. Analysen zum zeitund diskursgeschichtlichen Kontext ihres Schreibens, die Erforschung des intertextuellen Horizonts ihrer Werke, komparatistische und interdisziplinäre Untersuchungen, die durch Bachmanns vielfältige Kontakte und Lektüren und nicht zuletzt durch ihre Grenzüberschreitungen zwischen Literatur, Philosophie, Psychologie und Musik begründet sind, aber auch die historischkritische Revision der verfügbaren Nachlaßtexte tragen ebenso zu diesem Umbruch bei wie der literaturwissenschaftliche Methodenwandel, der neue Lektürerahmen geschaffen hat. Zugleich ist die Textgrundlage in den letzten Jahren sukzessiv durch neue Quellenfunde und -publikationen ergänzt worden, die nicht zuletzt das Wissen über die Breite der schriftstellerischen Tätigkeit Ingeborg Bachmanns, ihre literarische Arbeitsweise und die konkreten Entstehungskontexte ihrer Texte vertiefen. $\mathrm{Zu}$ nennen sind hier beispielsweise die frühe lyrische Prosa Briefe an Felician (1991), die kritische Edition des Todesarten-Projekts (1995), die u.a. auch bis dahin unbekannte Nachlaßfragmente wie den ersten Todesarten-Roman und den Goldmann/Rottwitz-Roman enthält, die Wiederentdeckung der bis dahin verschollenen Römischen Reportagen (1998) für Radio Bremen und die »Westdeutsche Allgemeine Zeitung «, die Edition »Letzter, unveröffentlichter Gedichte« (1998) sowie weiterer bislang unveröffentlichter Gedichtentwürfe aus den 1960er Jahren unter dem Titel »Ich weiß keine bessere Welt« (2000). Inzwischen ist im Nachlaß von Jörg Mauthe, Bachmanns Kollegen aus ihrer Zeit bei dem amerikanischen Besatzungssender RotWeiß-Rot (1951-1953) ein Teil von Bachmanns Beiträgen zu der Sendereihe Die Radiofamilie aufgefunden worden (McVeigh 2002), und es ist zu hoffen, daß weitere Nachlaßpublikationen und Quellenfunde folgen. Darüber hinaus liefern $\mathrm{Zi}$ tate aus dem unveröffentlichten Nachlaß und aus diversen Korrespondenzen wertvolle neue Hinweise zum Verständnis von Leben und Werk, und das vorliegende Handbuch fügt hier noch einiges hinzu. Solche Editionen und Neuentdeckungen ergänzen nicht nur die Materialgrundlage der literarhistorischen Forschung, sie eröffnen auch neue Fragestellungen und werden zweifellos eine wichtige Rolle in der weiteren Entwicklung des Bildes von Ingeborg Bachmann in Wissenschaft und Öffentlichkeit spielen. Gleichwohl ist »das Desiderat einer kritischen Gesamtausgabe des Werks von Ingeborg Bachmann" (Bartsch 2000, S. 373) zu bekräftigen. 
Es gehört zu den Glücksfällen der Literaturwissenschaft, daß das wissenschaftliche Interesse am Werk Ingeborg Bachmanns und das offenbar anhaltende Interesse einer breiten Leserschaft sich seit der 'Wiederentdeckung des Werks in den frühen 1980er Jahren gegenseitig befruchtet habẹn. Gleichzeitig hat die Funktion von Bachmanns Werk als Kristallisationspunkt aktueller literaturwissenschaftlicher Methodendiskussion - insbesondere auch weiterhin im Bereich des Feminismus, und nicht nur im deutschsprachigen Raum, sondern auch in der amerikanischen und französischen Germanistik - zu einer Forschungsproduktivität geführt, die heute für den einzelnen kaum noch vollständig zu überschauen ist. Vor diesem Hintergrund faßt das vorliegende Bachmann-Handbuch, das sowohl für die Bachmannforschung und die Literaturwissenschaft allgemein als auch für eine interessierte Leserschaft konzipiert wurde, das gewachsene Wissen über das Werk der Autorin auf neuestem Stand zusammen, zieht in lesbarer Form eine kritische Bilanz der Forschung (und ihrer Lücken) und stellt darüber hinaus neue Erkenntnisse und Interpretationsperspektiven vor. Die einzelnen Artikel versuchen also einer doppelten Aufgabenstellung gerecht zu werden: Sie sollen den Leserinnen und Lesern in zuverlässiger Form den Wissens- und Forschungsstand zu dem jeweiligen Werkkomplex oder Thema erschließen, zugleich aber auch die Forschung durch neue Einsichten und eigene Akzentuierung vorantreiben. Das Team der Autorinnen und Autoren setzt sich aus etablierten und jüngeren Bachmann-Forscherinnen und -Forschern zusammen, die ihren jeweils unterschiedlichen Ansätzen entsprechend auch verschiedene Perspektivierungen einbringen. Es muß bei einem solchen auf Pluralität angelegten Konzept nicht eigens betont werden, daß die vorgetragenen Positionen nicht notwendig mit denen der Herausgeber zusammenfallen.

Das Handbuch hat selbstverständlich die Funktion eines Nachschlagewerks, es soll aber auch zu neuer Werklektüre anregen und der Forschung neue Impulse geben. In diesem Sinne ist es nach dem einleitenden Überblick über Leben und Werk, Rezeptions- und Editionsgeschichte - in zwei Teile gegliedert, deren erster das Werk aus der Perspektive der Werkgruppen und Einzelwerke (und damit auch werkgeschichtlich) er- schließt, während der zweite ergänzend relevante Kontexte und Diskurse in Bachmanns Werk aufbereitet, die bei einer einzelwerkbezogenen Betrachtung nicht ausreichend gewürdigt werden können.

Ein so umfangreiches Projekt wie dieses Handbuch kommt nicht ohne vielfältige Zusammenarbeit und Unterstützung zustande. In diesem Sinne danken wir in erster Linie den Autorinnen und Autoren der Beiträge für ihre intensive Mitarbeit und den Erben Ingeborg Bachmanns (Isolde Moser und Dr. Heinz Bachmann) für die freundliche Erlaubnis zur Verwendung bislang unveröffentlichter Nachlaß- und Briefzitate. Unser Dank gilt hier zugleich den Archiven, aus deren Bestand zitiert werden durfte - der Österreichischen Nationalbibliothek (Wien), dem Deutschen Literaturarchiv (Marbach/N.), dem Archiv der Akademie der Künste (Berlin), dem Literaturarchiv Monacensia (München), dem Archiv des Südwestdeutschen Rundfunks (Stuttgart), den Verlagsarchiven Suhrkamp und Piper, dem Heinrich Böll Archiv (Köln) und dem Uwe Johnson Archiv (Frankfurt/M.) -, sowie Robert Pichl für die Benutzung seines Katalogs der Privatbibliothek Ingeborg Bachmanns (Pichl 2003). Unserem Lektor Uwe Schweikert schließlich danken wir für die kontinuierliche Förderung des Projekts von der ersten Anregung bis zur Drucklegung.

Abschließend noch einige Hinweise zur Benutzung des Handbuchs: Ergänzend zum Inhaltsverzeichnis erschließen ein Personen- und ein Werkregister die Gegenstände des Handbuchs. Der Anhang enthält darüber hinaus ein Literaturverzeichnis mit den relevanten Ausgaben der Werke Ingeborg Bachmanns sowie einer Auswahl der Literatur über die Autorin und ihr Werk. Mit Bezug auf dieses Literaturverzeichnis wird in den einzelnen Artikeln in abgekürzter Form zitiert: Werke Ingeborg Bachmanns werden unter Verwendung der im Ausgabenverzeichnis erläuterten Siglen zitiert; die im Anhang aufgeführten Drucke und Ausgaben werden im Literaturverzeichnis der Einzelartikel nicht nochmals aufgeführt. Sekundärliteratur aus dem Anhang wird im Literaturverzeichnis der Einzelartikel nur in abgekürzter Form nach dem Muster »McVeigh (2002)《 zitiert und den ausführlichen bibliographischen Angaben zu weiterer Sekundärliteratur vorangestellt. 
Literatur: McVeigh (2002); Pichl (2003).

Kurt Bartsch (2000): Rezension [Bachmann 2000b, Albrecht/Göttsche 2000, Weigel 1999]. In: Sprachkunst 31, S. 371-380; - Günter Blöcker (1954): 'Lyrischer Schichtwechsel ‘. In: Süddeutsche Zeitung, 13./14. November 1954; - Inge Stephan, Regula Venske, Sigrid
Weigel (1987): Die Literatur von Frauen vor der Frauenliteratur. Vorbemerkung. In: Stephan, Venske, Weigel: Frauenliteratur ohne Tradition? Neun Autorinnenporträts. Frankfurt/M., S. 7-9.

Monika Albrecht und Dirk Göttsche 